\title{
Joint reconstruction of activity and attenuation in non-TOF PET using a synergistic prior to enforce structural similarities
}

\author{
Ludovica Brusaferri, Student Member, IEEE, Elise C. Emond, Student Member, IEEE, David Atkinson, \\ Sébastien Ourselin, Brian F. Hutton, Senior Member, IEEE, Simon Arridge \\ and Kris Thielemans, Senior Member, IEEE
}

\begin{abstract}
This study investigates the use of a synergistic edgepreserving prior for a maximum-likelihood reconstruction of PET activity and attenuation images. The effect of the prior was tested on different reconstruction methods: MLAA and our algorithm MLAA-EB-S. Simple simulations on cylindrical phantoms with an insert showed that the presence of a synergistic prior enforces cross-talk related edges when the images are reconstructed with non-TOF MLAA and increases the bias in both the reconstructed activity and attenuation images. However, benefits appear when the joint problem is less ill-posed, as in the case of our method MLAA-EB-S, which exploits scatter information to improve conditioning. Overall, MLAA-EB-S with synergistic prior achieved the lowest bias and variance in both the activity and the attenuation images amongst the compared methods. Therefore, the proposed synergistic prior could be a useful way for improving the performance of MLAA-EB-S without the need of relying on anatomical images.
\end{abstract}

\section{INTRODUCTION}

$\mathbf{P}$ ET attenuation correction (AC) can be challenging in both PET/MR and PET/CT. In the case of PET/CT scanners, errors can be present because of misregistration between the PET and CT, as a result of a non-simultaneous acquisition between the two modalities [1]. In clinical PET/MR scanners, synthetic CT images are created from MR images with methods such as segmentation, atlas/mapping techniques or MR/CT learning. These approaches can be problematic in the thorax and the upper abdomen, because of the large variability of lung attenuation values and the presence of motion [2].

Joint reconstruction of activity and attenuation represents a promising method to overcome these limitations. One approach is to iteratively solve the inverse problem using likelihood optimisation (MLAA) [3]. However, without time-offlight the problem is very ill-conditioned, especially in lowdose acquisitions. A prior encouraging mu-values around bone, soft-tissue and lung-tissue is often used in joint reconstruction problems [3]. However, a population-based lung-tissue prior might not be appropriate given the considerable variation in lung-density, especially in patients with lung diseases such as Idiopathic Pulmonary Fibrosis.

In this work, we explore a different approach that relies on the expectation that the reconstructed attenuation and activity images are likely to share image structures. We exploit this

Ludovica Brusaferri was supported by Siemens Healthineers, the UCL Impact Scheme, and the EPSRC-funded UCL Centre for Doctoral Training in Medical Imaging (EP/L016478/1). Elise Emond was supported by GlaxoSmithKline (BIDS3000030921). We also acknowledge funding by the NIHR UCLH Biomedical Research Centre.

L. Brusaferri, B. F. Hutton and K. Thielemans are with the Institute of Nuclear Medicine, UCL, London, UK.). D. Atkinson is with the Centre for Medical Imaging, UCL, London, UK. S. Arridge is with Department of Computer Science, UCL, London, UK. S. Ourselin is with the Department of Medical Physics and Biomedical Engineering, KCL, London, UK. by incorporating a synergistic prior into the joint reconstruction framework, similar to previous synergistic PET and MR reconstructions [4]-[7] and Spectral CT (see [8] for some references).

\section{Methods}

\section{A. Synergistic Prior}

Here we investigate the incorporation of a synergistic edgepreserving regulariser, the Joint Total Variation (JTV). It favours sparsity in the gradient domain and therefore encourages common edges between the two unknown distributions. This prior is defined as:

$$
\mathrm{JTV}=\beta \int\left(\alpha^{2}+|\nabla \lambda(x)|^{2}+|\nabla \mu(x)|^{2}\right)^{1 / 2} \mathrm{~d} x
$$

where $\lambda(x)$ and $\mu(x)$ denote the activity and attenuation images respectively, $x \in \mathbb{R}^{3}, \beta$ is the global strength of the prior and $\alpha$ is a smoothing parameter introduced to avoid nondifferentiability.

\section{B. Reconstruction Algorithms}

We tested the effect of the proposed prior with two different reconstruction methods: MLAA [3] and our algorithm MLAAEB-S [9], which uses data from two energy windows and accounts for the photon energy information. Our implementation of both algorithms relies on L-BFGS-B [10] to simultaneously update $\lambda$ and $\mu$ to optimise the cost-function.

\section{EXPERIMENTS AND RESULTS}

\section{A. Simulated Phantom Data}

Experiments were conducted on a 3D cylindrical phantom with an insert (Fig. 1). Activity and attenuation image values were in the same scale, although with different contrast. We present results for the case when the attenuation and activity distributions have matching edges (Fig. 1, a-d) and when they do not (Fig. 1, e-h). The initial activity image estimates (Fig. $1, \mathrm{~d}, \mathrm{~h})$ were obtained by iterating 3 times between OSEM and SSS [11]. Data were simulated by forward projecting the ground truth activity image into sinograms corresponding to those obtained from the Siemens mMR. We used an energy resolution of $2 \%$ to assess the performance of the algorithms in ideal conditions. Poisson noise was added to the simulated data. 


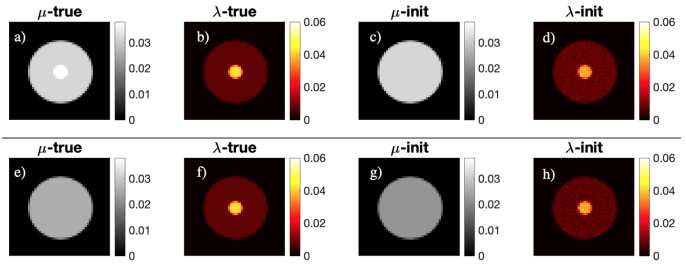

Fig. 1: From left to right: true attenuation $(\mathrm{a}, \mathrm{e})$, true activity $(b, f)$, initial attenuation $(\mathrm{c}, \mathrm{g})$, initial activity $(\mathrm{e}, \mathrm{h})$. First row: matching edges. Second row: unmatching edges.

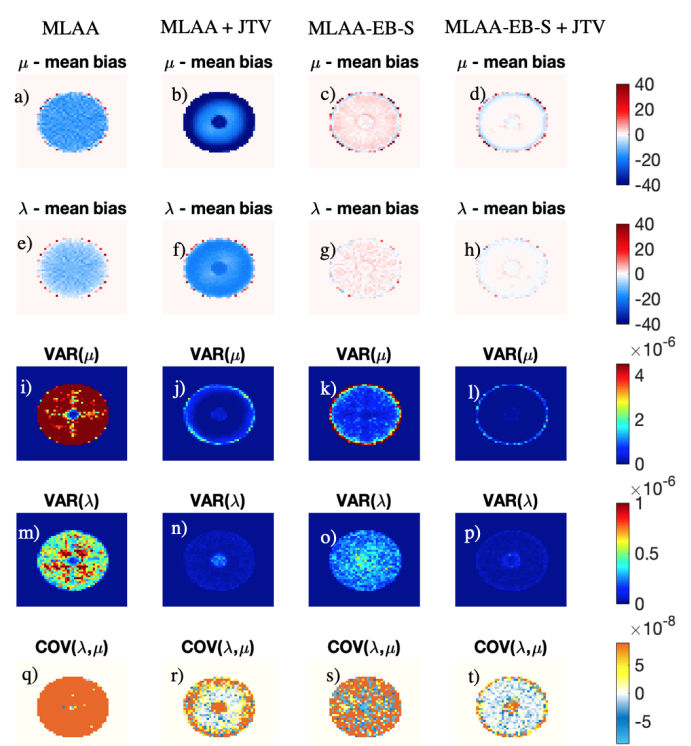

Fig. 2: First and second rows: bias images. Third and fourth rows: variance images. Fifth row: covariance images. Each column refers to a different reconstruction algorithm.

\section{B. Prior Parameter and Metrics}

We chose a smoothing factor corresponding to $2 \%$ of the background value in the attenuation image. The global penalty strength $(\beta=0.1)$ was deliberately chosen quite high, to emphasise the effects of the prior. Fine-tuning of the prior was not the purpose of this study. 30 noise realisations were used to compute the (voxel-wise) mean bias, variance and covariance images. For a numerical assessment, we computed the ROImeans in the insert for each metric, reported in Table I for the case of matching edges.

\section{Results}

Cross-talk appears, as expected, in both the case of matching and unmatching edges when images are reconstructed with MLAA (Fig. 2 and Fig. 3, a, e). The presence of the synergistic prior dramatically increases the bias in the reconstructed images (Fig. 2 and Fig. 3 b, f), however reducing both the variance and covariance (Fig. 2 j, n, f).

The MLAA-EB-S algorithm is able on its own to reconstruct the images with lower bias with respect to MLAA (Fig. 2 and Fig. 3, c, g). Here the presence of a synergistic prior benefits the reconstruction, further improving the stability of the algorithm, by reducing the variance (see Table I). For both the case of MLAA+JTV and MLAA-EB-S+JTV a global reduction of the covariance in the background comes at the cost of a higher covariance in the insert. For MLAA-EB-S, we observed a slightly increased bias in the case of matching

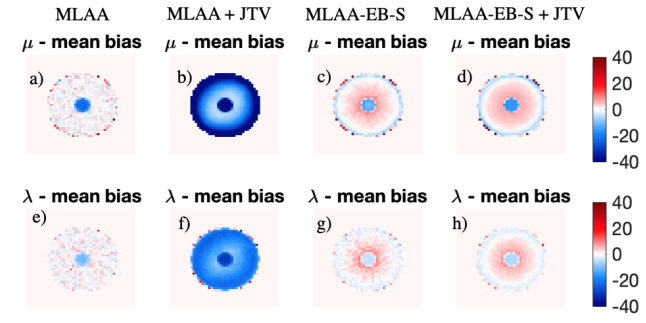

Fig. 3: First and second rows: bias images for the four reconstruction algorithms.

edges (Fig. $2 \mathrm{~d}, \mathrm{~h}$ ), probably due to an excessive weight of the prior. Variance and covariance have a similar trend in both the case of matching and unmatching edges - they are shown for one case only for reason of space.

TABLE I: ROI-means in the insert (unmatching edges). Background values: $\mu^{\mathrm{bg}}=0.032 \mathrm{~cm}^{-1}, \lambda^{\mathrm{bg}}=0.0084 \mathrm{a}$.u. Insert: $\mu^{\text {in }}=0.042 \mathrm{~cm}^{-1}, \lambda^{\text {in }}=0.042$ a.u.

\begin{tabular}{|c|c|c|c|c|}
\hline & MLAA & MLAA + JTV & MLAA-EB-S & MLAA-EB-S + JTV \\
\hline BIAS $(\mu)$ & $-15.01 \%$ & $-34.36 \%$ & $-1 \%$ & $-0.80 \%$ \\
$\operatorname{BIAS}(\lambda)$ & $-10.33 \%$ & $-20.86 \%$ & $-0.15 \%$ & $-0.12 \%$ \\
$\operatorname{VA} R(\mu)$ & $2.45 \mathrm{e}-06$ & $3.90 \mathrm{e}-07$ & $4.17 \mathrm{e}-07$ & $8.88 \mathrm{e}-08$ \\
$\operatorname{VA} R(\lambda)$ & $4.15 \mathrm{e}-07$ & $2.08 \mathrm{e}-07$ & $3.71 \mathrm{e}-07$ & $9.42 \mathrm{e}-08$ \\
$\operatorname{CO} \mathrm{O} V(\lambda, \mu)$ & $1.49 \mathrm{e}-07$ & $4.22 \mathrm{e}-07$ & $4.43 \mathrm{e}-09$ & $6.41 \mathrm{e}-08$ \\
\hline
\end{tabular}

\section{Discussion AND CONCLUSION}

We investigated the benefits of incorporating a synergistic prior into MLAA and our algorithm MLAA-EB-S.

The main finding of this study is that presence of the prior interacts with cross-talk during reconstruction, differently to synergistic PET/MR reconstruction where the two images are reconstructed from different data-sets. For MLAA, the presence of the prior enforces cross-talk related edges in the reconstructed images. The bias in the estimated activity increases from $-10.33 \%$ (MLAA) to $-20.86 \%$ (MLAA + JTV). In contrast, when multiple energy window information is used, the presence of the synergistic prior seems to provide benefit, possibly because to the cross-talk between the two estimated images is weaker: MLAA-EB-S + JTV achieved a final error in the activity image of $-0.12 \%$, with the lowest variance amongst all the four methods.

The matching edges experiments follow the same trend. However, we observed a higher bias in MLAA-EB-S + JTV compared to the case of unmatching edges. We hypothesise that this might be due to effects of initialisation and it will be the object of further investigation.

Overall, the incorporation of a synergistic prior seems promising for MLAA-EB-S but it is discouraged for a MLAAtype reconstruction (for non-TOF data), as it increases crosstalk.

\section{REFERENCES}

[1] A. Martinez-Moller et al., J Nucl Med, vol. 48, no. 2, 2007.

[2] J. Lillington et al., Medical Physics, 2019.

[3] J. Nuyts et al., IEEE Trans Med Im, vol. 18, no. 5, 1999.

[4] M. J. Ehrhardt et al., Inverse Problems, vol. 31, 2015.

[5] F. Knoll et al., IEEE Trans Med Im, vol. 36, no. 1, 2017.

[6] A. Mehranian et al., IEEE Trans Med Im, vol. 37, no. 1, 2018.

[7] - Magn Reson Med, vol. 81, no. 3, 2019.

[8] D. S. Rigie et al., Phys in Med and Biol, vol. 60, 2015.

[9] L. Brusaferri et al., NSS/MIC Conf. Proc. [accepted], 2018.

[10] R. H. Byrd et al., SIAM J. Sci. Comput., vol. 16, no. 5, 1995.

[11] C. C. Watson et al., 3D Im Rec. in Rad. and Nucl. Med., 1996. 